\title{
Vascular and Interventional Radiology Workflow Management During the COVID-19 Pandemic: Position Statement by the Saudi Interventional Radiology Society
}

\section{Preface}

Coronavirus disease pandemic (COVID-19) is caused by a novel virus from the Coronavirus family. The virus was identified on December 31, 2019, as a cause of pneumonia in Wuhan City, China. It was labeled as COVID-19 on January 7, 2020.

The disease is transmitted from human to human through droplet with a high communicability causing a rapid spread. On January 26, 2020, the World Health Organization (WHO) categorized the global risk from COVID-19 as high. By March 11, 2020, the WHO declared COVID-19 as a pandemic. ${ }^{[1]}$

On January 21, 2020, the Government of Saudi Arabia started to take precautionary measures to control COVID-19 and limit its spread to ensure the safety of its citizens and residents. Through collaboration of several governmental bodies (The Ministry of Health $[\mathrm{MOH}]$, The National Center for Disease Prevention and Control, The Saudi Patient Safety Center, The Ministry of Interior, The Ministry of Municipal and Rural Affairs, The Ministry of Human Resources and Social Development, Medical Societies, etc.), progressive measures and decisions have been implemented including:

- Suspension of travels to and from countries where COVID-19 is endemic on January 26, 2020. On March 15, 2020, all international flights have been suspended, followed by domestic flights and transportation suspension on March 21,2020

- Suspension of public presence in all sport competitions and games on March 5, 2020, followed by suspension of all sports activities and shutting down

This is an open access journal, and articles are distributed under the terms of the Creative Commons Attribution-NonCommercial-ShareAlike 4.0 License, which allows others to remix, tweak, and build upon the work non-commercially, as long as appropriate credit is given and the new creations are licensed under the identical terms.

For reprints contact: WKHLRPMedknow_reprints@wolterskluwer.com private gyms and sport centers on March 14, 2020

- Suspension of Umrah, then prayers in mosques to prevent gathering on March 4, 2020, and March 17, 2020, respectively

- Suspension of public and private schooling on March 8, 2020

- A daily curfew from 7:00 pm to 6:00 am for 21 days starting from March 23, 2020 .

The first reported case in Saudi Arabia was on March 2, 2020, for a citizen arriving from Iran. Until the day of this document (March 22, 2020), there are 511 reported cases, 17 of which have recovered, and no deaths.

As healthcare institutions remain at the frontline during such an outbreak, highest levels of preventive and precautionary measures must be taken to ensure staff and public safety. Vascular and Interventional Radiology (VIR) as a specialty represents a fundamental part of this framework and plays an essential role in its integrity. VIR covers a wide spectrum of disease pathologies, ranging from emergency to elective procedures. In addition, it interconnects several departments in the healthcare system, which can potentially result in unnecessary exposure to both staff and patients. As the influx of critically ill patients may increase, VIR teams will need to adjust workflow to minimize elective procedures and accommodate more emergency interventions. Therefore, the Saudi Interventional Radiology Society (SIRS) is offering this guide to better manage VIR preparedness and workflow during outbreaks, such as COVID-19.

How to cite this article: Ashour MA, Arabi M, Justaniah Al. Vascular and interventional radiology workflow management during the COVID-19 pandemic: Position statement by the Saudi Interventional Radiology Society. Arab J Interven Radiol 2020;4:67-72.

\section{Majed A. Ashour ${ }^{1}$, Mohammad Arabi², Almamoon I. Justaniah $^{3}$}

${ }^{1}$ President, Saudi Interventional Radiology Society, Department of Radiology, Division of Vascular and Interventional Radiology, King Faisal Specialist Hospital and Research Center, Jeddah, ${ }^{2} \mathrm{Head}$, Research Committee, Saudi Interventional Radiology Society, Department of Medical Imaging, Division of Vascular and Interventional Radiology, Ministry of National Guard Health Affairs, King Abdullah International Medical Research Center, King Saud Bin Abdulaziz University for Health Sciences, Riyadh, ${ }^{3}$ Vice President, Saudi Interventional Radiology Society, Head, Scientific Committee, Saudi Interventional Radiology Society, Department of Medical Imaging and Intervention, King Abdullah Medical City, Makkah, Saudi Arabia

Received: 24-03-2020 Accepted: 26-03-2020 Online Published: 28-03-2020

Address for correspondence: Dr. Majed A. Ashour, President, Saudi Interventional Radiology Society.

E-mail: ashourma@hotmail.com

Access this article online

Website: www.arabjir.com

DOI: 10.4103/AJIR.AJIR_5_20

Quick Response Code:

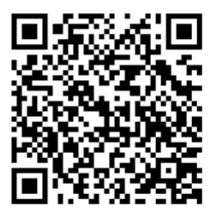




\section{Hospital Preparedness}

This section is covered by the Saudi $\mathrm{MOH}^{[2]}$, $\mathrm{WHO}^{[1]}$, and the Center of Disease Control and Prevention (CDC).$^{[3]}$ However, the society recommends that hospitals should:

- Initiate a COVID-19 team to deal with suspected and confirmed COVID-19 cases, in addition to providing daily updates and guidance

- Ensure proper staff education regarding infection control measures, hand hygiene, N-95 mask fitting, and careful donning and doffing of appropriate personal protective equipment (PPE)

- Maintain adequate supply of PPE.

\section{Patient Care}

- Cancel outpatient VIR clinics and reschedule patients according to their medical needs

- Instruct patients to call the VIR coordinator should their condition necessitates further attention.

- Maintain a list of canceled patients to reschedule them once the outbreak resolves to ensure proper follow-up

- Cease all research activities that involve patients visiting the hospital solely for research purposes

- Minimize mobilization and transfer of COVID-19 patients to VIR suites for procedures and perform bedside procedures whenever possible.

\section{Healthcare Workers}

Healthcare workers include physicians (interventional neuroradiologists, vascular interventional radiologists, and nonvascular interventional radiologists), nurses, technologists, trainees, and administrative personnel.

- Assign a team leader for daily two-way communication with the hospital administration to receive updates and plan accordingly

- Move reporting workstations from reading rooms to offices

- Maintain enough workforce according to your institutional needs

- Create separate teams, at least two, with a 1-week on to 1-week off rotations. Each team should be able to function independently to avoid cross coverage. Ensure no physical interaction between the on-site team and the off-site team

- Halt all unnecessary meetings and switch the crucial ones from physical to virtual platforms

- Perform virtual morning rounds or limit staff involvement to the minimum requirement while maintaining social distancing

- Use standard precautions and proper PPE with suspected or positive COVID-19 patients

- Practice social distancing and hand hygiene at all times

- Refer to the Saudi MOH, CDC, and WHO guidelines for proper use of PPE.

\section{Visitors}

- Stop visitors from accessing the VIR recovery

- Prevent families and friends from accompanying patients to the VIR department.

\section{Supply}

Optimization of resources utilization is a requisite to maintain services sustainability. As suppliers are shutting down and travels are suspended, proper allocation of resources is important to ensure availability of these resources when needed.

- Maintain adequate supply of PPE within the department

- Plan and maintain adequate supply of VIR materials within the department

- Preserve critical and low supply items to critical cases

- Optimize utilization of materials and use the minimum requirement when possible.

\section{Procedure Room}

- Designate a VIR suite/procedure room with negative pressure for COVID-19 patients when possible

- Use the standard precaution and follow the guidelines for the periprocedural room and equipment preparation and cleaning

- Limit the number of people involved in the procedure to the minimum safe requirement.

\section{Procedures}

In daily practice, balancing between patient care and optimal utilization of resources is imperative. With patient safety in mind, appropriate scheduling of the patients and procedures according to their clinical urgency can be challenging. This becomes more apparent during crisis and outbreaks. The Saudi Patient Safety Center (SPSC) proposed a list of categories to prioritize all surgical and medical interventions performed in Saudi Arabia in collaboration with the corresponding societies [Table 1]. Therefore, SIRS has created the following priority list as guide and reference to the interventional radiologists to better prioritize their patients/procedures [Tables 2-6]. This list was endorsed by the SPSC and is accessible at (https://spsc.gov.sa/Arabic/pages/guidelines.aspx).

In addition, the society recommends to:

Table 1: Categories of procedure priority according to the Saudi Patient Safety Center

\begin{tabular}{|c|c|}
\hline $\begin{array}{l}\text { Priority } \\
\text { category }\end{array}$ & Definition \\
\hline Priority 1 & Emergent: Intervention is required within $24 \mathrm{~h}$ \\
\hline Priority 2 & Urgent: Intervention is required within 7 days \\
\hline Priority 3 & Nonurgent: Intervention is required within 30 day \\
\hline Priority 4 & $\begin{array}{l}\text { Elective/routine: Intervention can be performed } \\
\text { after } 30 \text { days }\end{array}$ \\
\hline
\end{tabular}


Table 2: Prioritization of vascular, nonvascular, and neurointerventional procedures according to disease category

\begin{tabular}{|c|c|c|}
\hline Category of disease & Vascular and interventional procedures/cases & Priority \\
\hline Aortic/Arterial & $\begin{array}{l}\text { Angiogram with/without embolization for acute bleeding (abdominal, bronchial } \\
\text { pulmonary, GI, postpartum, etc.) }\end{array}$ & 1 \\
\hline Aortic/arterial & Embolization of peripheral/visceral AVM & 4 \\
\hline Aortic/arterial & Endovascular management of renal artery stenosis & 4 \\
\hline Aortic/arterial & Endovascular management of aortic/arterial aneurysm (asymptomatic) & 4 \\
\hline Aortic/arterial & Endovascular management of aortic/arterial pseudoaneurysm & 1 \\
\hline Aortic/arterial & Endovascular management of aortic/vascular injury/trauma & 1 \\
\hline Aortic/arterial & Endovascular management of aortic aneurysm (ruptured) & 1 \\
\hline Aortic/arterial & Endovascular management of aortic aneurysm (symptomatic or large) & 2 \\
\hline Aortic/arterial & Endovascular management of limb ischemia (acute) & 1 \\
\hline Aortic/arterial & Endovascular management of limb ischemia (critical) & 2 \\
\hline Aortic/arterial & Endovascular management of limb ischemia (noncritical) & 3 \\
\hline Venous & Central or peripheral venous lines & 2 \\
\hline Venous & Embolization of female pelvic varices & 4 \\
\hline Venous & Embolization of scrotal varicocele & 4 \\
\hline Venous & Endovascular management of acute DVT & 2 \\
\hline Venous & Endovascular management of acute pulmonary embolism & 1 \\
\hline Venous & Endovascular management of chronic venous occlusion & 4 \\
\hline Venous & Endovascular management of dialysis fistula, acute occlusion & 2 \\
\hline Venous & Endovascular management of dialysis fistula, malfunctioning & 3 \\
\hline Venous & Intravascular foreign body retrieval & 1 \\
\hline Venous & IVC filter insertion & 2 \\
\hline Venous & IVC filter retrieval & 3 \\
\hline Venous & Management of varicose veins (endovenous ablation/sclerotherapy) & 4 \\
\hline Venous & Portal vein recanalization & 4 \\
\hline Venous & Temporary dialysis catheter insertion for acute renal failure/leukopheresis & 1 \\
\hline Venous & TIPSS/BRTO for variceal bleeding & 1 \\
\hline Venous & TIPSS for ascites & 4 \\
\hline Venous & Transjugular liver biopsy/pressure measurement & 3 \\
\hline Venous & Tunneled dialysis catheter insertion for ESRD & 2 \\
\hline Venous & Venous sampling (adrenal, renal, petrosal, etc.) & 4 \\
\hline Venous/lymphatic & Management of low flow malformation (venous/lymphatic) & 4 \\
\hline Lymphatic & Lymphatic thoracic duct embolization & 3 \\
\hline Oncology & Benign tumor, percutaneous ablation (thyroid, bone, etc.) & 4 \\
\hline Oncology & Benign tumors, embolization (uterine fibroids, prostate enlargement, renal AML, etc.) & 4 \\
\hline Oncology & Malignant tumor ablation/chemoembolization/radioembolization (liver, renal, lung, etc.) & 3 \\
\hline Oncology & Portal vein embolization & 3 \\
\hline Body/nonvascular & Airway/GI stenting (acute obstruction) & 2 \\
\hline Body/nonvascular & Airway/GI stenting (nonobstructing) & 3 \\
\hline Body/nonvascular & Fallopian tubes recanalization for infertility & 4 \\
\hline Body/nonvascular & Percutaneous antegrade ureteral stenting & 4 \\
\hline Body/nonvascular & Percutaneous biliary drainage for biliary obstruction & 2 \\
\hline Body/nonvascular & Percutaneous biliary drainage for biliary obstruction (septic cholangitis) & 1 \\
\hline Body/nonvascular & Percutaneous drainage of abdominal/pleural collection & 2 \\
\hline Body/nonvascular & Percutaneous drainage of abdominal/pleural/others collection (septic) & 1 \\
\hline Body/nonvascular & Percutaneous insertion of feeding tubes & 3 \\
\hline Body/nonvascular & Percutaneous insertion of tunneled peritoneal or pleural catheters & 3 \\
\hline Body/nonvascular & Percutaneous needle biopsy (solid organs, lungs, thyroid, bone, etc.) & 3 \\
\hline Body/nonvascular & Percutaneous needle biopsy of transplanted solid organs (liver, renal) & 2 \\
\hline Body/nonvascular & Percutaneous nephrostomy for renal obstruction & 2 \\
\hline Body/nonvascular & Percutaneous nephrostomy for renal obstruction (septic) & 1 \\
\hline Body/nonvascular & Tube change (biliary, nephrostomy, gastrostomy, cecostomy, etc.) & 4 \\
\hline Neuro & Endovascular assessment of epilepsy & 4 \\
\hline
\end{tabular}




\begin{tabular}{|c|c|c|}
\hline \multicolumn{3}{|c|}{ Table 2: Contd... } \\
\hline Category of disease & Vascular and interventional procedures/cases & Priority \\
\hline Neuro & Endovascular management of acute intracranial aneurysm rupture & 1 \\
\hline Neuro & Endovascular management of acute intracranial AVM rupture & 2 \\
\hline Neuro & Endovascular management of acute stroke & 1 \\
\hline Neuro & Endovascular management of asymptomatic intracranial AVM/aneurysm/dural fistula & 4 \\
\hline Neuro & Endovascular management of carotid artery stenosis (asymptomatic) & 4 \\
\hline Neuro & Endovascular management of carotid artery stenosis (symptomatic) & 3 \\
\hline Neuro & Endovascular management of carotid/vertebral dissection (asymptomatic) & 3 \\
\hline Neuro & Endovascular management of carotid/vertebral dissection (with hemorrhage or stroke) & 1 \\
\hline Neuro & Fluoroscopy-guided lumbar puncture & 2 \\
\hline Neuro & Management of CNS tumors (ablation, embolization, chemoinfusion) & 3 \\
\hline Pain management & Facet, nerve root, joint injections, and plexus block/neurolysis & 4 \\
\hline Pain management & Percutaneous vertebroplasty/kyphoplasty for spinal fractures/tumors & 4 \\
\hline \multicolumn{3}{|c|}{$\begin{array}{l}\text { TIPSS: Transjugular intrahepatic portosystemic shunt, BRTO: Balloon-occluded retrograde transvenous obliteration, DVT: Deep } \\
\text { venous thrombosis, CNS: Central nervous system, GI: Gastrointestinal, AVM: Arteriovenous malformation IVC: Inferior vena cava, } \\
\text { ESRD: End-stage renal disease, AML: Angiomyolipoma }\end{array}$} \\
\hline \multicolumn{3}{|c|}{ Table 3: Priority 1 interventions (emergent intervention is required within $24 \mathrm{~h}$ ) } \\
\hline Category of disease & Vascular and interventional procedures/cases & Priority \\
\hline Aortic/arterial & $\begin{array}{l}\text { Angiogram with/without embolization for acute bleeding (abdominal, bronchial } \\
\text { pulmonary, GI, postpartum, etc.) }\end{array}$ & 1 \\
\hline Aortic/arterial & Endovascular management of aortic/arterial pseudoaneurysm & 1 \\
\hline Aortic/arterial & Endovascular management of aortic/vascular injury/trauma & 1 \\
\hline Aortic/arterial & Endovascular management of aortic aneurysm (ruptured) & 1 \\
\hline Aortic/arterial & Endovascular management of limb ischemia (acute) & 1 \\
\hline Venous & Endovascular management of acute pulmonary embolism & 1 \\
\hline Venous & Intravascular foreign body retrieval & 1 \\
\hline Venous & Temporary dialysis catheter insertion for acute renal failure/leukopheresis & 1 \\
\hline Venous & TIPSS/BRTO for Variceal bleeding & 1 \\
\hline Body/nonvascular & Percutaneous biliary drainage for biliary obstruction (septic cholangitis) & 1 \\
\hline Body/nonvascular & Percutaneous drainage of abdominal/pleural/others collection (septic) & 1 \\
\hline Body/nonvascular & Percutaneous nephrostomy for renal obstruction (septic) & 1 \\
\hline Neuro & Endovascular management of acute intracranial aneurysm rupture & 1 \\
\hline Neuro & Endovascular management of acute stroke & 1 \\
\hline Neuro & Endovascular management of carotid/vertebral dissection (with hemorrhage or stroke) & 1 \\
\hline
\end{tabular}

TIPSS: Transjugular intrahepatic portosystemic shunt, BRTO: Balloon-occluded retrograde transvenous obliteration, GI: Gastrointestinal

\begin{tabular}{|c|c|c|}
\hline Category of disease & Vascular and interventional procedures/cases & Priority \\
\hline Aortic/arterial & Endovascular management of aortic aneurysm (symptomatic or large) & 2 \\
\hline Aortic/arterial & Endovascular management of limb ischemia (critical) & 2 \\
\hline Venous & Central or peripheral venous lines & 2 \\
\hline Venous & Endovascular management of acute DVT & 2 \\
\hline Venous & Endovascular management of dialysis fistula, acute occlusion & 2 \\
\hline Venous & IVC filter Insertion & 2 \\
\hline Venous & Tunneled dialysis catheter insertion for ESRD & 2 \\
\hline Body/nonvascular & Airway/GI stenting (acute obstruction) & 2 \\
\hline Body/nonvascular & Percutaneous biliary drainage for biliary obstruction & 2 \\
\hline Body/nonvascular & Percutaneous drainage of abdominal/pleural collection & 2 \\
\hline Body/nonvascular & Percutaneous needle biopsy of transplanted solid organs (liver, renal) & 2 \\
\hline Body/nonvascular & Percutaneous nephrostomy for renal obstruction & 2 \\
\hline Neuro & Endovascular management of acute intracranial AVM rupture & 2 \\
\hline Neuro & Fluoroscopy-guided lumbar puncture & 2 \\
\hline
\end{tabular}

DVT: Deep venous thrombosis, IVC: Inferior vena cava, ESRD: End-stage renal disease, AVM: Arteriovenous malformation 
Table 5: Priority 3 interventions (nonurgent intervention is required within $\mathbf{3 0}$ days)

\begin{tabular}{llc}
\hline Category of disease & Vascular and interventional procedures/cases & Priority \\
\hline Aortic/arterial & Endovascular management of limb ischemia (noncritical) & 3 \\
Venous & Endovascular management of dialysis fistula, malfunctioning & 3 \\
Venous & IVC filter retrieval & 3 \\
Venous & Transjugular liver biopsy/pressure measurement & 3 \\
Lymphatic & Lymphatic thoracic duct embolization & 3 \\
Oncology & Malignant tumor ablation/chemoembolization/radioembolization (liver, renal, lung, etc.) \\
Oncology & Portal vein embolization & 3 \\
Body/nonvascular & Airway/GI stenting (nonobstructing) \\
Body/nonvascular & Percutaneous insertion of feeding tubes \\
Body/nonvascular & Percutaneous insertion of tunneled peritoneal or pleural catheters \\
Body/nonvascular & Percutaneous needle biopsy (solid organs, lungs, thyroid, bone, etc.) \\
Neuro & Endovascular management of carotid artery stenosis (symptomatic) \\
Neuro & Endovascular management of carotid/vertebral dissection (asymptomatic) \\
Neuro & Management of CNS tumors (ablation, embolization, chemoinfusion) \\
\hline
\end{tabular}

CNS: Central nervous system, GI: Gastrointestinal IVC: Inferior vena cava

Table 6: Priority 4 interventions (elective/routine intervention can be performed after 30 days)

\begin{tabular}{ll}
\hline Category of disease & Vascular and interventional procedures/cases \\
\hline Aortic/arterial & Embolization of peripheral/visceral AVM \\
Aortic/arterial & Endovascular management of renal artery stenosis \\
Aortic/arterial & Endovascular management of aortic/arterial aneurysm (asymptomatic) \\
Venous & Embolization of female pelvic varices \\
Venous & Embolization of scrotal varicocele \\
Venous & Endovascular management of chronic venous occlusion \\
Venous & Management of varicose veins (endovenous ablation/sclerotherapy) \\
Venous & Portal vein recanalization \\
Venous & TIPSS for ascites \\
Venous & Venous sampling (adrenal, renal, petrosal, etc.) \\
Venous/lymphatic & Management of low flow malformation (venous/lymphatic) \\
Oncology & Benign tumor, percutaneous ablation (thyroid, bone, etc.) \\
Oncology & Benign tumors, embolization (uterine fibroids, prostate enlargement, renal AML, etc.) \\
Body/nonvascular & Fallopian tubes recanalization for infertility \\
Body/nonvascular & Percutaneous antegrade ureteral stenting \\
Body/nonvascular & Tube change (biliary, nephrostomy, gastrostomy, cecostomy, etc.) \\
Neuro & Endovascular assessment of epilepsy \\
Neuro & Endovascular management of asymptomatic intracranial AVM/aneurysm/dural fistula \\
Neuro & Endovascular management of carotid artery stenosis (asymptomatic) \\
Pain management & Facet, nerve root, joint injections, and plexus block/neurolysis \\
Pain management & Percutaneous vertebroplasty/kyphoplasty for spinal fractures/tumors \\
\hline
\end{tabular}

TIPSS: Transjugular intrahepatic portosystemic shunt, AVM: Angiomyolipoma

- Limit procedures to inpatients and ambulatory priority category 1 and 2

- Limit interventions to patients whom intervention may affect their management/outcome

- Perform bedside procedures when possible for COVID-19 patients

- Use your clinical judgment for patients under priority category 3

- Reschedule patients under priority category 4.

\section{Future Directions}

This pandemic may change how medicine is going to be practiced across the globe. It may affect businesses, hospitals' design, patients' triage, etc. Developing a healthcare system and infrastructure that can function smoothly during crisis is imperative. Telemedicine and virtual meetings represent an excellent advantageous surrogate of a helpful infrastructure.

Financial support and sponsorship

Nil.

Conflicts of interest

There are no conflicts of interest. 


\section{References}

1. World Health Organization. Coronavirus Disease COVID-19 Data. Available from: https://www.who.int/emergencies/diseases/ novel-coronavirus-2019. [Last accessed on 2020 Mar 22].
2. The Saudi Ministry of Health. Available from: https://www.moh. gov.sa. [Last accessed on 2020 Mar 22].

3. Saudi Press Agency. Available from: https://www.spa.gov.sa. [Last accessed on 2020 Mar 22]. 